\title{
An Efficient Photoelectrochemical Hydrogen Evolution System using Silicon Nanomaterials with Ultra-High Aspect Ratios
}

\author{
Duck Hyun Lee, ${ }^{*[a]}$ Sai P. R. Kobaku,${ }^{[b]}$ Young-Rae Hong ${ }^{[c]}$ and Jae Young Kwon ${ }^{[a]}$
}

We fabricated ultra-high aspect ratio silicon nanomaterials, including a silicon nanomesh and silicon nanowire array, on a wafer scale for efficient photoelectrochemical hydrogen production. These silicon nanomaterials (feature size $\approx 20 \mathrm{~nm}$ ) possess a high aspect ratio to increase the optical absorptivity of the cells to approximately $95 \%$ over a broad range of wavelengths. The silicon nanomesh and Si nanowire cells achieved high photocurrent values of 13 and $28 \mathrm{~mA} \mathrm{~cm}^{-2}$, respectively, which are increased by $200 \%$ and
$570 \%$ in comparison to their bulk counterparts. In addition, these scalable Si nanomaterials remained stable for up to $100 \mathrm{~min}$ of hydrogen evolution. Detailed studies on the doping and geometrical structures of the resulting hydrogen evolution cells suggest that both the $\mathrm{n}^{+} \mathrm{pp}^{+}$doping and thickness of nanostructures are keys to the enhancement of the hydrogen evolution efficiency. The results obtained in this work show that these silicon nanomaterials can be used for high-performance water-splitting system applications.

\section{Introduction}

Energy harvesting directly from sunlight has been a desirable approach toward producing clean energy with minimal environmental impact. ${ }^{[1-3]}$ Conversion of solar energy by photoelectrolysis of water using semiconductors and then subsequently storing the hydrogen in simple molecular form is a highly desirable approach to solve the energy challenge. ${ }^{[4-9]}$ To accomplish the efficient photoelectrolysis of water, the choice of suitable photoelectrode materials is of high importance. The properties of the photoelectrode, such as optical absorption, carrier transportation, and chemical stability, determine the performance of the photoelectrochemical cell. ${ }^{[5-7,9,10]}$ Among the various photoelectrode materials, silicon is an attractive candidate for a photocathode because it possesses both a suitable band gap for efficient sunlight absorption as well as an appropriate position of the conduction band with respect to the electrochemical potential of water reduction. ${ }^{[5,10,11]}$ The main challenge in developing Si-based photocathode materials is to simultaneously achieve high light absorption and efficient charge carrier collection. Recently, the utilization of micro- and nanostructured Si electrodes has attracted attention for solar energy conversion. ${ }^{[7,9,10,12-14]}$ Compared to a planar electrode with the same direction of light absorption and charge carrier collection, the use of nanostructured electrodes allows the directions of light absorption and charge carrier collection to be decoupled while still offering the potential for high energy conversion efficiency. ${ }^{[15,16]}$ Moreover, the nanostructured electrode can significantly increase light absorption by increasing optical path length. ${ }^{[17-20]}$ Thus, the preparation of Si nanostructures of high aspect ratio and high areal density over a large area can be a breakthrough for a realizing a high-performance photoelectrochemical water splitting system.
In this study, we developed precisely controlled, Si nanomaterials (nanomesh and nanowires) of ultra-high aspect ratio on a wafer scale by using the combination of block copolymer lithography and metal-assisted etching. Block copolymer lithography is an emerging approach that provides a variety of periodic nanoscale morphologies having feature sizes ranging from 5 to $50 \mathrm{~nm} \cdot{ }^{[21-24]}$ Unlike other nanopatterning techniques such as focused electron-beam lithography ${ }^{[25]}$ or scanning probe lithography, ${ }^{[26]}$ block copolymer lithography provides a parallel patterning process, thereby enabling easy scale-up. In the metal-assisted Si etching method, a thin layer of noble metal on the surface of the Si substrate catalyzes the etching of $\mathrm{Si}$ in a mixed solution containing $\mathrm{HF}$ and $\mathrm{H}_{2} \mathrm{O}_{2}$, resulting in parallel pores or columnar structures on the (100) Si substrate. ${ }^{[12,13,27-29]}$ Metal-assisted Si etching is a simple, fast, low-cost and easily scaled-up technique, and various approaches have been derived previously to fabricate Si-based nanostructures. ${ }^{[12,13,29]}$

\footnotetext{
[a] Dr. D. H. Lee, Dr. J. Y. Kwon

Department of Materials Science and Engineering

University of Michigan

Ann Arbor, MI (USA)

E-mail:duckhyun.lee@umich.edu

[b] S. P. R. Kobaku

Department of Macromolecular Science Q Engineering

University of Michigan

Ann Arbor, MI (USA)

[c] Dr. Y.-R. Hong

Silicium Energy

Sunnyvale, CA (USA)

Supporting information for this article is available on the WWW under http://dx.doi.org/10.1002/ente.201402074
} 


\section{Results and Discussion}

A schematic illustration of the fabrication processes of the $\mathrm{Si}$ nanomesh is shown in Figure 1 a. The polystyrene (PS) nanoporous template was prepared by self-assembly of a block copolymer spin-coated onto a p-Si wafer. The Au catalytic thin film $(20 \mathrm{~nm})$ was deposited onto the PS nanoporous template and the subsequent lift-off process produced hexagonally packed Au catalytic nanoparticles over the entire p-Si substrate surface (Figure $1 \mathrm{~b}$ ). The average diameter and pitch of the Au nanoparticles were $20 \mathrm{~nm}$ and $34 \mathrm{~nm}$, respectively. The prepared substrate was dipped into the $6 \mathrm{~m} \mathrm{HF} /$ $0.6 \mathrm{M} \mathrm{H}_{2} \mathrm{O}_{2}$ aqueous solution for metal-assisted etching. ${ }^{[27,28]}$ Upon etching, holes were generated due to the preferential reduction of the oxidant on the surface of the Au nanoparticles, and the holes diffused through the Au nanoparticles, injecting into the Si substrate. The Si was oxidized by the injected holes and dissolved at the $\mathrm{Si} / \mathrm{Au}$ nanoparticles interface by HF. This process enabled the etching of the p-Si substrate downward and led to vertically aligned uniform nanopores within the Si substrate. ${ }^{[27,28]}$ The resulting Si nanomesh had hexagonally packed and very uniform nanopores over the $\mathrm{p}$-Si substrate surface with a nanopore diameter of $24 \mathrm{~nm}$ (Figure 1c). The pitch of the nanopores and the thinnest width between the nanopores within the $\mathrm{p}$-Si nanomesh were $34 \mathrm{~nm}$ and $10 \mathrm{~nm}$, respectively. The etched nanopores ran straight down, parallel to the normal direction of Si surface (Figure $1 \mathrm{~d}$ ); after $10 \mathrm{~min}$ of etching, the Au nanoparticles a

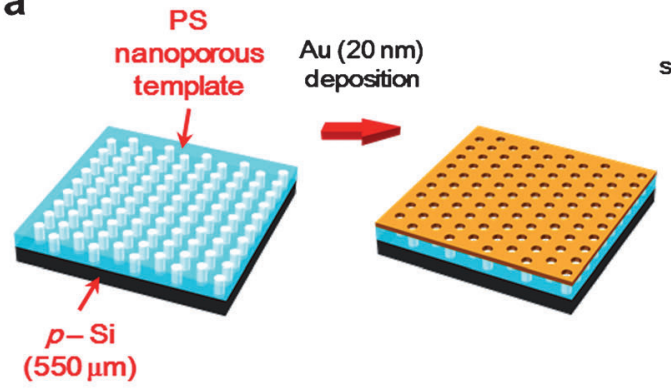

b

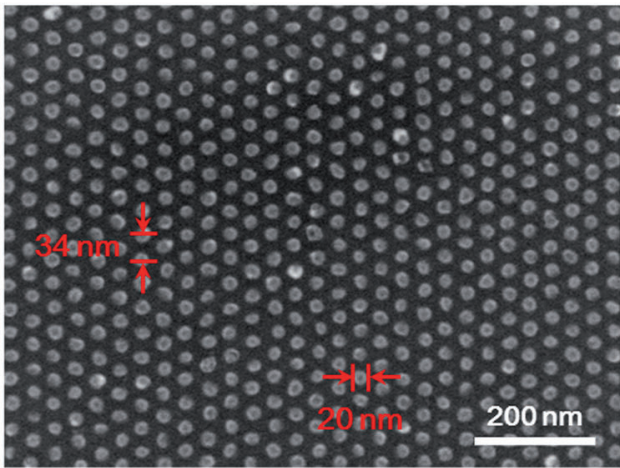

d

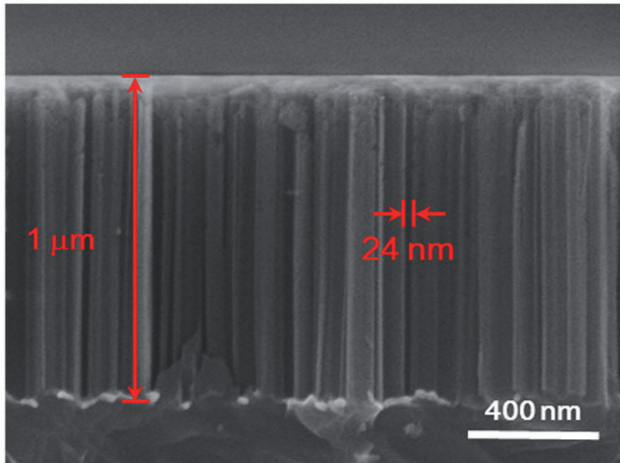

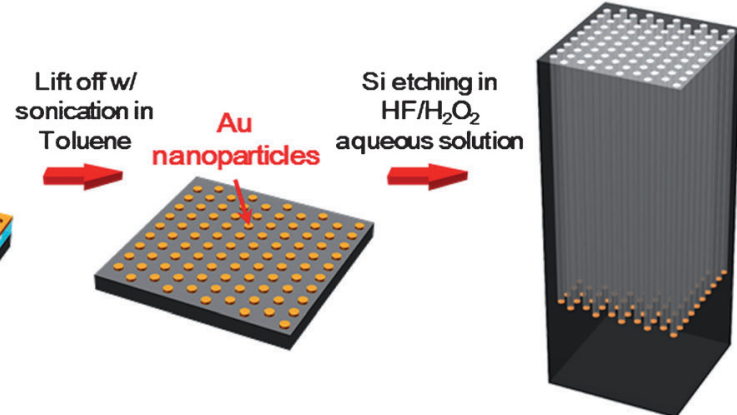

C

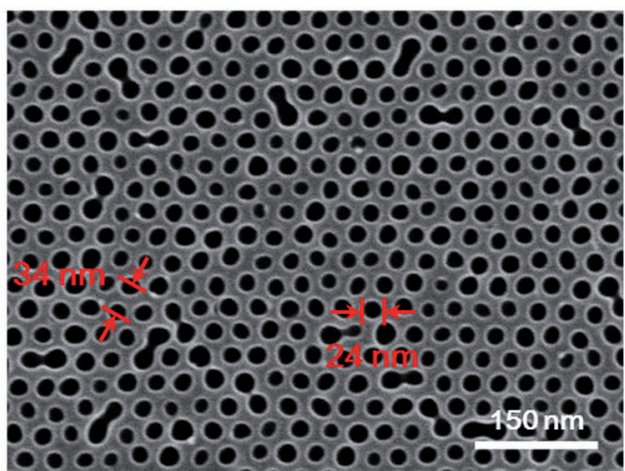

e

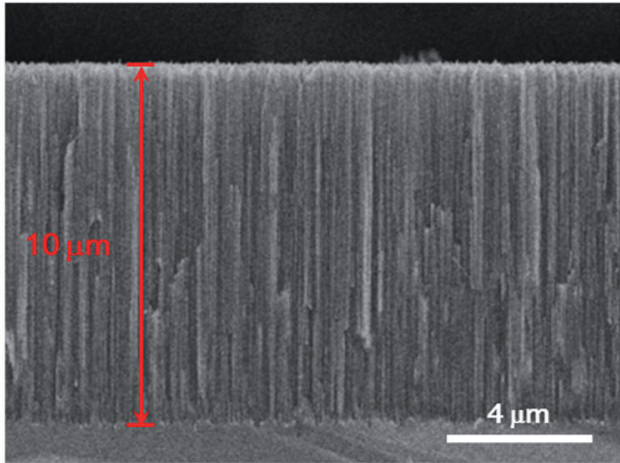

Figure 1. a) Schematic illustration of the Si nanomesh fabrication process. b) Plan-view SEM image of the catalytic Au nanoparticles after lift-off process. The diameter and pitch of the hexagonally packed Au nanoparticles were $20 \mathrm{~nm}$ and $34 \mathrm{~nm}$, respectively. c) Plan-view SEM image of the Si nanomesh after $20 \mathrm{~s}$ of metal-assisted etching. The image shows the hexagonal packing of the etched nanopores. The diameter and pitch of the nanopores within the nanomesh were $24 \mathrm{~nm}$ and $34 \mathrm{~nm}$, respectively. d) and e) Cross-sectional SEM images of Si nanomesh after $1 \mathrm{~min}$. (d) and $10 \mathrm{~min}$ (e) of Au-assisted etching. These images show that Si substrate is etched vertically and the diameter of the etched nanopores $(24 \mathrm{~nm})$ was maintained uniformly all along the etching direction. The $10 \mathrm{~min}$ of metal-assisted etching produced a $10 \mu \mathrm{m}$ long nanopores array, which resulted in an aspect ratio of 420 . 
etched nanopores approximately $10 \mu \mathrm{m}$ deep into the Si substrate (Figure 1e, also see Supporting Information Figure $\mathrm{S} 1)$.

All the process steps for the preparation of the Si nanowires are similar to those of Si nanomesh (Figure $2 \mathrm{a}$ ). A thin Cr layer $(20 \mathrm{~nm})$ was deposited onto to the PS nanoporous template and the PS template was lifted off to prepare the hexagonally packed uniform $\mathrm{Cr}$ nanoparticles with high areal density over the p-Si substrate surface (see Supporting Information, Figure S2a). The hexagonal pattern of Cr nanoparticles was used as a mask for Au-assisted etching of the Si substrate. Before the deposition of the Au thin film, the p-Si substrate with $\mathrm{Cr}$ nanoparticles was slightly etched $(15 \mathrm{~nm})$ using reactive-ion etching (Figure $2 \mathrm{~b}$ ) to prevent the formation of a continuous Au layer. A thin Au film $(8 \mathrm{~nm})$ was deposited onto to the wafer, which provides a honeycombstructured $\mathrm{Au}$ film in contact with the Si surface, and the rest of the Au film is left on top of the Cr nanoparticles (see Supporting Information, Figure $\mathrm{S} 2 \mathrm{~b}$ ). The prepared substrate was dipped into the $6 \mathrm{M} \mathrm{HF} / 0.6 \mathrm{M} \mathrm{H}_{2} \mathrm{O}_{2}$ aqueous solution, and the thin $\mathrm{Au}$ film etched the Si downward leading to a vertically aligned uniform Si nanowire array over the entire substrate surface. For the fabrication of vertically aligned $\mathrm{Si}$ nanowires, it is critical to have a high density of $\mathrm{Cr}$ nanoparticles as the etching mask. The low density of $\mathrm{Cr}$ nanoparticles resulted in non-uniform Si etching, which eventually a

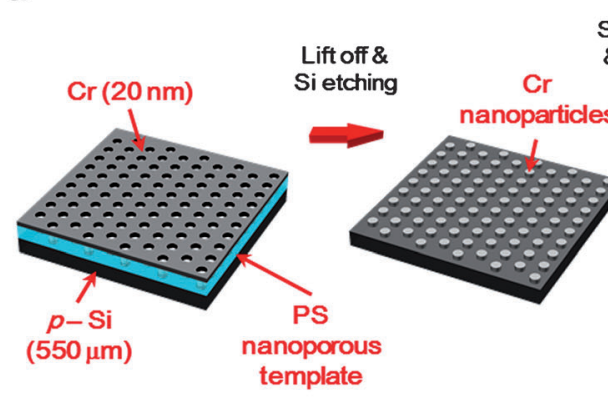

b

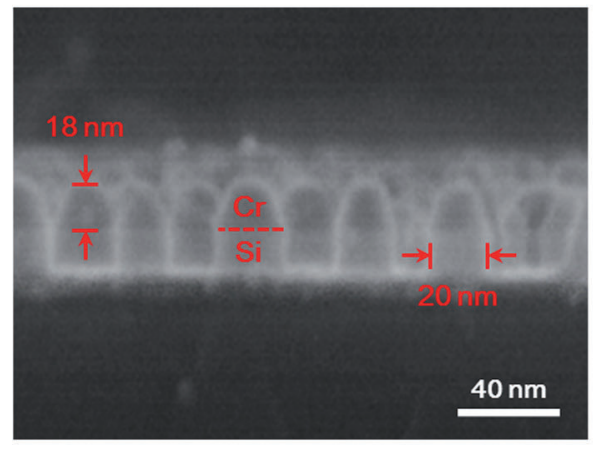

d

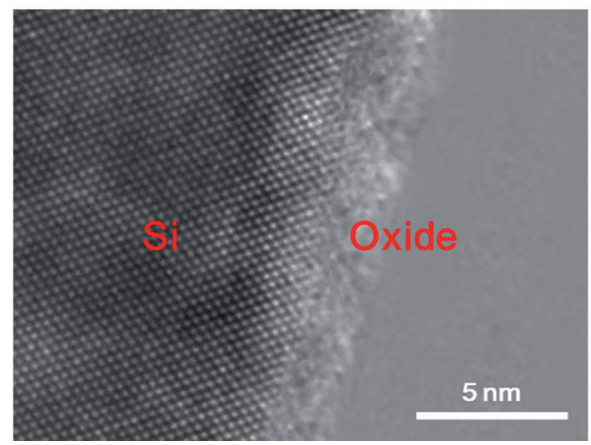

C

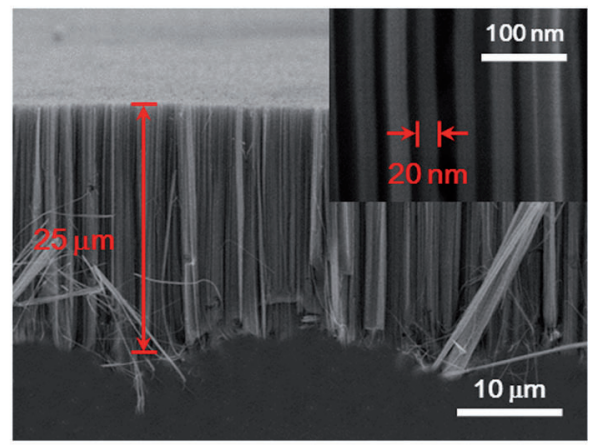

e

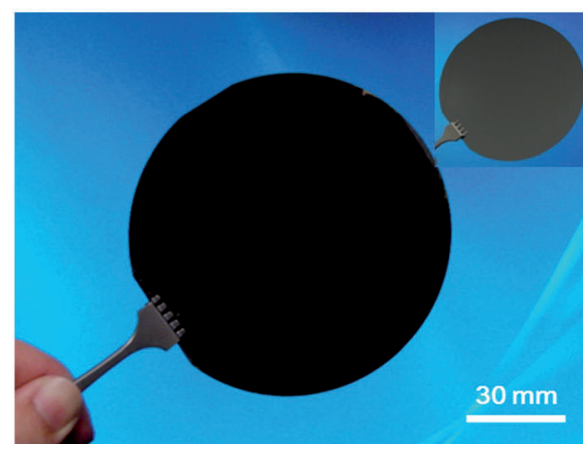

Figure 2. a) Schematic illustration of the Si nanowire array fabrication process. b) Cross-sectional SEM image of the Si nanopillars (height $\approx 15 \mathrm{~nm}$ ) with $\mathrm{Cr}$ nanoparticles on top of the pillars obtained after lift-off and the Si etching step. c) Cross-sectional SEM image of the prepared Si nanowire array after 25 min of Au-assisted etching. The diameter and the length of the Si nanowire were $20 \mathrm{~nm}$ and $25 \mu \mathrm{m}$, respectively, which resulted in an aspect ratio of 1250. The inset is a high-magnification SEM image of the Si-nanowire array showing that the nanowires remain separated. d) High-resolution TEM image of a segment of a Si nanowire. The roughness at the interface between the crystalline silicon and the amorphous native oxide is observed. e) Optical image of Si nanowire array over an entire 4-inch Si wafer. The inset image shows a bulk 4-inch Si wafer. The dark appearance of the Si wafer with nanowire array as compared to the bulk Si wafer indicates that the Si nanowire array surface is non-reflective due to light scattering by the Si nanowire array.

would lead to the collapse of the nanowires (see Supporting Information, Figure S2 c). After 25 min of etching, the vertically aligned uniform $\mathrm{Si}$ nanowire array were prepared with an approximate length of $25 \mu \mathrm{m}$ (see Supporting Information, Figure S1). After drying the Si nanowire array, all of the $\mathrm{Si}$ nanowires remained separate (Figure 2c). The diameters of the prepared $\mathrm{Si}$ nanowires were very uniform, and the mean diameter of $\mathrm{Si}$ nanowire was $20 \mathrm{~nm}$ on top and $22 \mathrm{~nm}$ on bottom (see Supporting Information, Figure S3 a).

The TEM image of the Si nanowires (Figure $2 \mathrm{~d}$ ) indicates the formation of a native oxide ( $\approx 3 \mathrm{~nm}$ thickness) around the single-crystalline $\mathrm{Si}$ nanowire lattice. The surfaces of the $\mathrm{Si}$ nanowires were very rough (see Supporting Information, Figure S3b), which may be attributed to the randomness of the lateral oxidation and etching in the etching solution. ${ }^{[29]}$ As the all the steps involved in the fabrication process, (the self-assembly of nanotemplates, $\mathrm{Au}$ catalyst deposition, and metalassisted etching) entail parallel processing, this approach can be readily scaled up to the preparation of a large-scale photoelectrochemical hydrogen evolution system. Figure $2 \mathrm{e}$ shows the $\mathrm{Si}$ nanowire array prepared on an entire 4-inch $(1$ inch $=2.54 \mathrm{~cm}) \quad$ wafer. The dark appearance of the wafer 
with the Si nanowire array in comparison to that of bulk $\mathrm{Si}$ wafer indicates that the Si nanowire array wafer is non-reflective due to light scattering by the nanowire array, and the rough surface may additionally increase the light absorption.

For the photoelectrochemical hydrogen evolution measurement of the prepared Si nanomesh and Si nanowire substrates, they were doped with phosphorous on the nanostructured side and boron on the other side. This process yields a $\mathrm{n}^{+} \mathrm{pp}^{+}$doping structure, which can efficiently separate the charge carriers excited upon light illumination. ${ }^{[8,10,30]}$ Also we deposited a discontinuous layer of Pt electrocatalyst to accelerate the hydrogen evolution reaction while maintaining reasonable photovoltages. ${ }^{[6,10,31]}$ Though there are multiple $\mathrm{Pt}$ deposition techniques, we used electroless deposition for its simplicity and low cost. The samples prepared in this way were immersed in an aqueous solution containing $0.1 \mathrm{~mm}$ $\mathrm{Na}_{2} \mathrm{PtCl}_{6} \cdot 6 \mathrm{H}_{2} \mathrm{O}$ and $0.5 \mathrm{M} \mathrm{HF}$ for $10 \mathrm{~min}$ at room temperature (see Supporting Information, Figure S4), and connected with metal wire for measurements.

Figure 3 a shows the representative current density vs. potential $J-E$ measurement curves under $70 \mathrm{~mW} \mathrm{~cm}^{-2}$ illumination using a tungsten-halogen white light source for three different cells: (1) $\mathrm{Pt} / \mathrm{n}^{+} \mathrm{pp}^{+}$plane Si cell, (2) $\mathrm{Pt} / \mathrm{n}^{+} \mathrm{pp}$ $1.1 \mu \mathrm{m}$ thick Si nanomesh cell, and (3) $\mathrm{Pt} / \mathrm{n}^{+} \mathrm{pp}^{+} 1.2 \mu \mathrm{m}$ long $\mathrm{Si}$ nanowire cell measured in aqueous $0.5 \mathrm{M} \mathrm{K}_{2} \mathrm{SO}_{4}$ solution. The solution was adjusted to $\mathrm{pH} 1$ with $\mathrm{H}_{2} \mathrm{SO}_{4}$, and the po- tential was measured by sweeping from positive to negative potentials with a scan rate of $50 \mathrm{mV} \mathrm{s}^{-1}$. The saturated current density $\left(J_{\text {sat }}\right)$ at $-0.8 \mathrm{~V}$ vs. reversible hydrogen electrode (RHE) for the plane Si cell, Si nanomesh cell, and Si nanowire cell were measured to be $3.9,12.6$, and $27.4 \mathrm{mAcm}^{-2}$, respectively. Thus the $\mathrm{Si}$ nanomesh and $\mathrm{Si}$ nanowire structures increased the photoelectrochemical hydrogen evolution efficiency by over $200 \%$ and $570 \%$, respectively, over their bulk counterparts. The current density $(J)$ measured in the dark remains approximately zero over the entire potential range, which indicates that the currents are generated from the light illumination. The photocurrent onset potentials $\left(E_{\text {onset }}\right)$ were defined as the potential at which the photocurrent and dark current merged in $J^{2}$ vs $V$ plots; and $E_{\text {onset }}$ was approximately $0.25 \mathrm{~V}$ for all three samples and changed negligibly. The significant increase of $J$ may be attributed to following mechanisms: (1) the absorbance enhancement caused by the light scattering in the $\mathrm{Si}$ nanomesh and $\mathrm{Si}$ nanowire array, ${ }^{[17-20]}$ (2) effective separation of the photogenerated charge carriers achieved along the transverse direction of the nanostructures due to the drastically reduced transport distance of the minority carriers, ${ }^{[15,16]}$ and (3) the nanostructured Si substrates possess a large surface area that greatly enhances water splitting as compared to its smooth bulk counterpart. $^{[5]}$

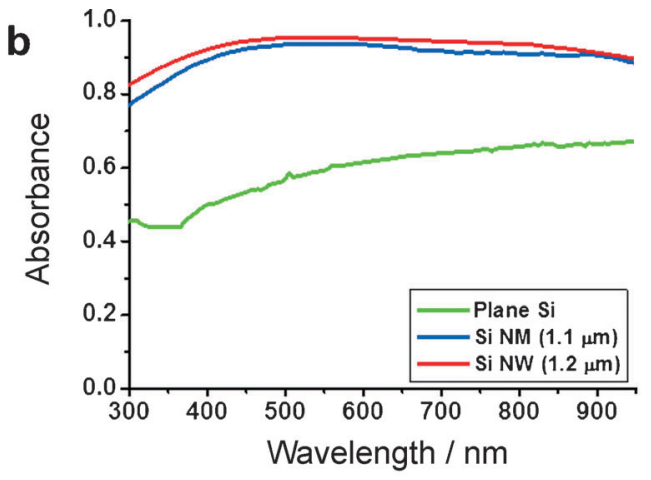

d

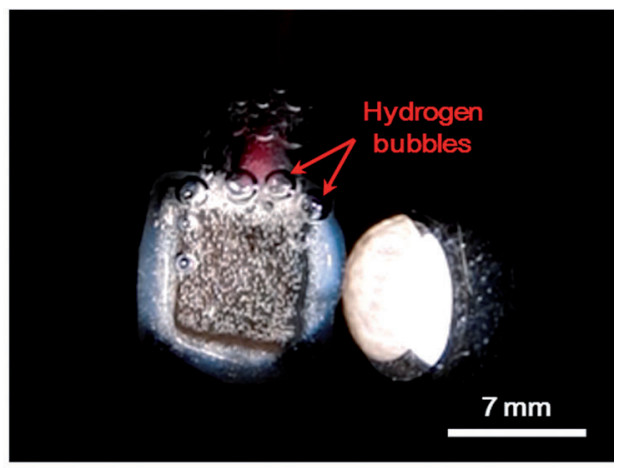

Figure 3. a) Representative current density vs. potential $(J-E)$ curves of the bare $\mathrm{p}$-Si (black), $1.1 \mu \mathrm{m}$ thick $\mathrm{Pt} / \mathrm{n}^{+} \mathrm{pp}^{+} \mathrm{Si}$ nanomesh (blue), and $1.2 \mu \mathrm{m}$ long Pt/ $\mathrm{n}^{+} \mathrm{pp}^{+}$Si nanowire (red) cells measured under a light intensity of $70 \mathrm{~mW} \mathrm{~cm}{ }^{-2}$ in aqueous $0.5 \mathrm{M} \mathrm{K}_{2} \mathrm{SO}_{4}$ solution adjusted to $\mathrm{pH}_{1}$ with $\mathrm{H}_{2} \mathrm{SO}_{4}\left(50 \mathrm{mV} \mathrm{s}{ }^{-1}\right.$, single sweep from positive to negative potentials). b) and c) Absorbance spectra (b) and external quantum efficiency spectra (c) for bare Si (black), $1.1 \mu \mathrm{m}$ thick Si nanomesh (blue), and $1.2 \mu \mathrm{m}$ long Si nanowire (red). d) Optical image of a normal $\mathrm{Pt} / \mathrm{n}^{+} \mathrm{pp}^{+}$silicon nanowire array electrode evolving hydrogen at $-0.8 \mathrm{~V}$ vs. $\mathrm{RHE}$ under $70 \mathrm{~mW} \mathrm{~cm}^{-2}$ illumination. Many small bubbles were nucleated on the Si nanowire surface, and further coalesced into large individual bubbles. 
To better elucidate the effects of Si nanomesh and Si nanowire structures on the absorbance $(A)$ of our photoelectrochemical hydrogen evolution cells, we measured the reflectance $(R)$ as a function of wavelength in the range of 300 $950 \mathrm{~nm}$ for the bare Si cell, $1.1 \mu \mathrm{m}$ thick Si nanomesh cell, and $1.2 \mu \mathrm{m}$ long Si nanowire cell. Figure $3 \mathrm{~b}$ shows the calculated absorbance curves for the three cells. The absorbance was calculated by subtracting the reflectance values from unity $(A=1-R)$ assuming the transmittance through $550 \mu \mathrm{m}$ of $\mathrm{Si}$ for wavelengths lower than $950 \mathrm{~nm}$ is negligible. ${ }^{[20]}$ It is clear (Figure $3 \mathrm{~b}$ ) that the Si nanomesh and Si nanowires dramatically enhanced the optical absorptivity to approximately $95 \%$ over a broad range of wavelength from visible to near IR. We also performed external quantum efficiency measurements in the visible and near-IR wavelengths for the three cells. Figure $3 \mathrm{c}$ shows the quantum efficiency curves for plane Si (black), the $1.1 \mu \mathrm{m}$ thick Si nanomesh cell (blue), and the $1.2 \mu \mathrm{m}$ long Si nanowire cell (red). At a wavelength of approximately $800 \mathrm{~nm}$, the plane Si cell exhibits approximately $15 \%$ quantum efficiency, whereas the quantum efficiency values for the $\mathrm{Si}$ nanomesh and $\mathrm{Si}$ nanowires reache $49 \%$ and $93 \%$, respectively, in agreement with the significant increase in $J_{\mathrm{SC}}$ caused by $\mathrm{Si}$ nanostructures (Figure $3 \mathrm{a}$ ). From the measurement, we can estimate that the Si nanowire converts approximately $98 \%\left(27.4 \mathrm{~mA} \mathrm{~cm}^{-2}\right)$ of the absorbed light to current for hydrogen evolution. Such high conversion rate is primarily due to the reduced transport distance for minority carriers and increased surface area, and it enabled a high hydrogen evolution rate (Figure $3 \mathrm{~d}$, also see Supporting Information Movie 1). However, the Si nanomesh only converts approximately $50 \%\left(12.6 \mathrm{~mA} \mathrm{~cm}^{-2}\right)$ of the observed light to current. This limitation may be due to the difficulty in transportation of the evolved hydrogen bubbles within the high-aspect-ratio nanopores of very small diameter $(24 \mathrm{~nm})$. The evolved hydrogen bubble can be captured within the nanopores, which decreases the effective surface area for the water reduction reaction.

To maximize the hydrogen evolution efficiency of photoelectodes, the doping structure and the geometrical structure are critical. We conducted detailed cell performance evaluations for five different photoelectrode cells to better understand the contribution of doping structure: $:^{[10,30]}$ (1) bare p-Si nanowire cell without Pt deposition, (2) Pt/bare p-Si nanowire cell, (3) Pt/pp ${ }^{+}$Si nanowire cell, (4) $\mathrm{Pt} / \mathrm{n}^{+} \mathrm{p}$ Si nanowire cell, and (5) $\mathrm{Pt} / \mathrm{n}^{+} \mathrm{pp}^{+} \mathrm{Si}$ nanowire cell. Here, we maintained the length of the Si nanowires to be $1.2 \mu \mathrm{m}$ for all the cells. Figure 4 a shows the $J-E$ curves for the five different cells under $70 \mathrm{~mW} \mathrm{~cm}^{-2}$ illumination. The bare $\mathrm{p}$-Si nanowires without $\mathrm{Pt}$ deposition showed almost negligible current density, whereas the $\mathrm{Pt} / \mathrm{n}^{+} \mathrm{pp}^{+}$Si nanowire cell showed the high-
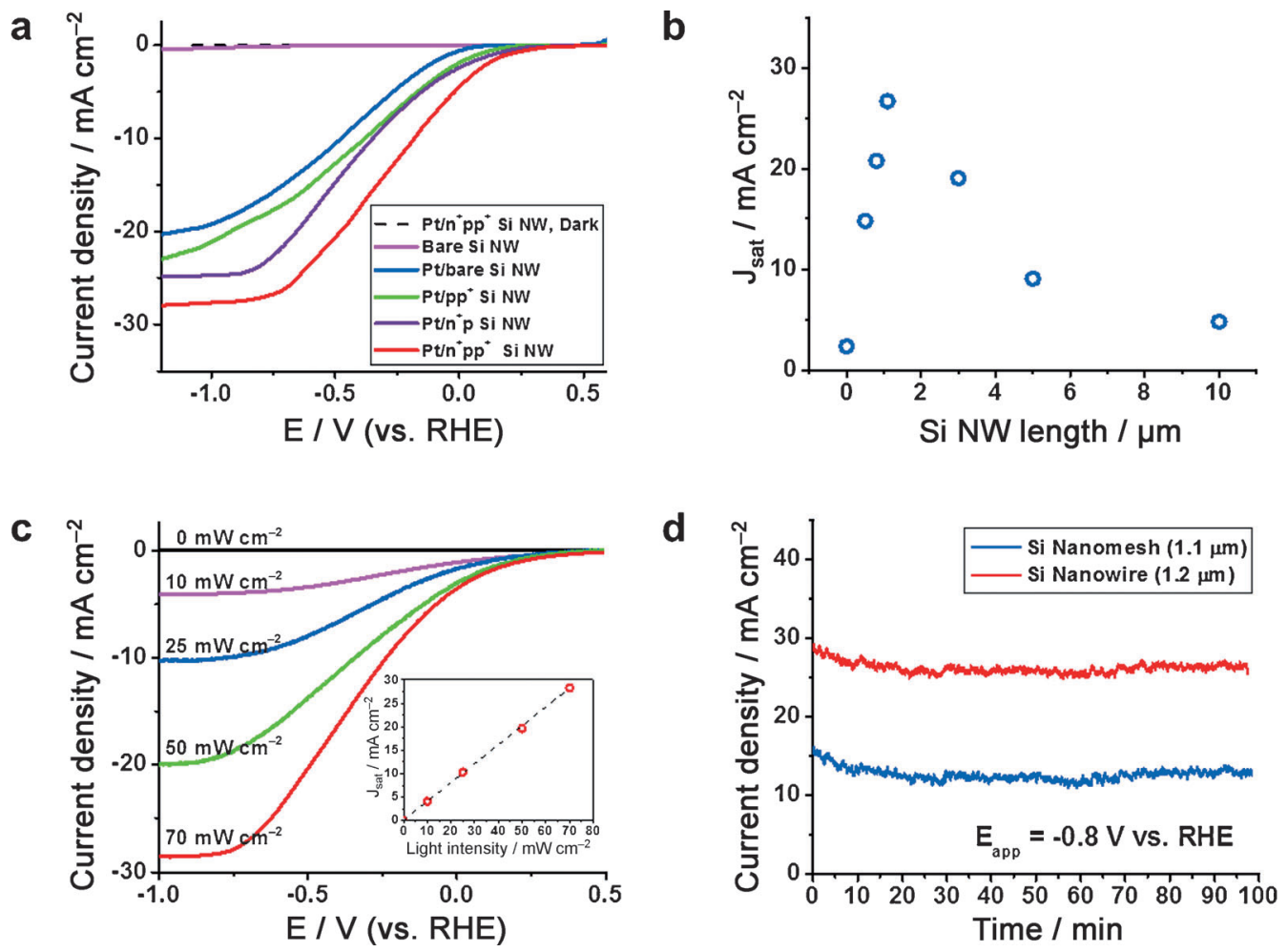

Figure 4. a) J--E curves of bare $\mathrm{p}$-Si nanowires (magenta), Pt/bare $\mathrm{p}$-Si nanowires (blue), $\mathrm{Pt} / \mathrm{pp}{ }^{+}$Si nanowires (green), Pt/n ${ }^{+} \mathrm{p} \mathrm{Si} \mathrm{nanowire} \mathrm{(purple),} \mathrm{and} \mathrm{Pt/}$ $\mathrm{n}^{+} \mathrm{pp}^{+} \mathrm{Si}$ nanowires cells under $70 \mathrm{mWcm}{ }^{-2}$ illumination. The average length of the Si nanowires is $1.2 \mu \mathrm{m}$. b) Saturated current density $\left(U_{\text {sat }}\right)$ as a function of the length of Si nanowires measured at $-0.8 \mathrm{~V}$ vs. RHE. All the electrodes were doped with the $\mathrm{n}^{+} \mathrm{pp}^{+}$structure and $70 \mathrm{~mW} \mathrm{~cm}^{-2}$ of light was illuminated. c) J-E curves of the Si-nanowires hydrogen evolution electrode at light intensities of $0,10,25,50$, and $70 \mathrm{~mW} \mathrm{~cm}^{-2}$. The inset compares the saturated current density $\left(J_{\text {sat }}\right)$ at $E=-0.8 \mathrm{~V}$ vs. RHE. d) Long-term current density $(J)$ measurement of the $1.1 \mu \mathrm{m} \mathrm{Pt} / \mathrm{n}^{+} \mathrm{pp}^{+} \mathrm{Si}_{\text {nanomesh }}(\mathrm{blue})$ and the $1.2 \mathrm{~mm} \mathrm{Pt} / \mathrm{n}^{+} \mathrm{pp} \mathrm{P}^{+} \mathrm{Si}$ nanowires (red) as a function of time measured during the hydrogen evolution at $-0.8 \mathrm{~V}$ vs. RHE. The current initially decreased by approximately $5 \%$ in the first 15 min before stabilizing. 
est current density $(J)$ and onset potential $\left(E_{\text {onset }}\right)$. This data clearly show that the $\mathrm{Pt}$ catalyst accelerates the hydrogen evolution reaction on the Si nanowire surface, and the combination of $n^{+}$doping on the frontside and $\mathrm{p}+$ doping on the backside efficiently separates the generated charge carriers as well as increases the onset potential of the photoelectrodes. These observations are in agreement with the results obtained from plane Si cells and Si nanomesh cells (see Supporting Information, Figure S5).

The saturated current density $\left(J_{\text {sat }}\right)$ as a function of $\mathrm{Si}$ nanowire length is presented in Figure $4 \mathrm{~b}$ as measured at $-0.8 \mathrm{~V}$ vs. RHE under $70 \mathrm{~mW} \mathrm{~cm}^{-2}$ illumination. All of the cells were prepared with the $\mathrm{Pt} / \mathrm{n}^{+} \mathrm{pp}^{+}$Si nanowire structure. The data shows that the $1.2 \mu \mathrm{m}$ long Si nanowire cell leads to the highest enhancement in current density. Interestingly, if the length of Si nanowire was higher than $3 \mu \mathrm{m}$, the current density decreased (also see Supporting Information, Figure S6). This behavior may be attributed to the morphology of $\mathrm{Pt}$ deposition. The electroless $\mathrm{Pt}$ deposition method forms an approximately $1 \mu \mathrm{m}$ deep, high-density $\mathrm{Pt}$ particle layer from the top end of Si nanowires (see Supporting Information, Figure S7). Therefore, if the length of the Si nanowire is approximately longer than $1 \mu \mathrm{m}$, the charge carriers generated from the light illumination near the bottom of the $\mathrm{Si}$ nanowires need to transfer to the top end of Si nanowire for hydrogen evolution, which may increase the chance of charge recombination.

To better understand the photoelectrochemical behavior of the $\mathrm{Pt} / \mathrm{n}^{+} \mathrm{pp}^{+} \mathrm{Si}$ nanowire photoelectrode, we measured the current density at various light intensities. Figure $4 \mathrm{c}$ shows the $J-E$ measurement curves under the light intensity of $0-70 \mathrm{~mW} \mathrm{~cm}^{-2}$. The inset compares the saturated current density $\left(J_{\text {sat }}\right)$ at $E=-0.8 \mathrm{~V}$ vs. RHE. as a function of light intensity, and it shows a linear relationship of current density and illumination power, which indicates that the Si nanowire photoelectrode has constant efficiency of charge separation under the condition of sufficient cathodic polarization. ${ }^{[32]} \mathrm{We}$ also ran the experiments for $100 \mathrm{~min}$ to confirm the stability of the hydrogen evolution with time on the Si nanomesh and Si nanowire electrodes. Figure $4 \mathrm{~d}$ shows the current density $(J)$ as a function of time measured during the hydrogen evolution at $E=-0.8 \mathrm{~V}$ vs. RHE under $70 \mathrm{~mW} \mathrm{~cm}^{-2}$ illumination. The current densities slightly decreased in the first $15 \mathrm{~min}$, however they produced stable current density and hydrogen evolution for up to $100 \mathrm{~min}$ of experimental run time.

\section{Conclusions}

The results from this work illustrate the successful fabrication of photoelectrochemical cells based on Si nanomesh and Si nanowire arrays with features as small as $20 \mathrm{~nm}$ with high aspect ratio. Thus Si-nanostructured cells were fabricated using scalable manufacturing techniques; they exhibited enhanced optical absorptivity up to $95 \%$, reduced minority carrier transport distance, and extremely high chemical reaction surface area. These structural advantages of the Si nanostruc- tures developed here enabled us to develop highly efficient photoelectrochemical hydrogen evolution cells with longterm stability. Detailed studies on the doping structure and geometrical structure of the Si nanomaterials further elucidated their effects on the overall hydrogen evolution efficiency. The results reported here may provide useful design considerations for developing a highly efficient photoelectrochemical water splitting system and other classes of solar energy conversion systems in the future.

\section{Experimental Section}

\section{Nanoporous polymer template preparation}

The (100) Si surface was neutrally treated with a random copolymer brush. A thin film $(100 \mathrm{~nm})$ of asymmetric block copolymer, polystyrene-block-poly(methyl methacylate) (PS-b-PMMA) that forms cylindrical nanostructures upon annealing (molecular weight: $P S / P M M A=46.1 \mathrm{k} / 21 \mathrm{k}$; PMMA cylinder diameter: $18 \mathrm{~nm}$, pitch distance between neighboring cylinders: $34 \mathrm{~nm}$ ) was spin-coated onto the wafer surface and annealed at $190^{\circ} \mathrm{C}$ for $24 \mathrm{~h}$. After annealing, the substrates were irradiated with ultraviolet light $(254 \mathrm{~nm})$ for $30 \mathrm{~min}$, and subsequently rinsed with acetic acid (30 $\mathrm{min})$ and water $(5 \mathrm{~min})$ to remove the PMMA cylinder cores. This left a nanoporous polystyrene template over the entire silicon surface.

\section{Fabrication of Si nanomesh electrode}

The substrates with polystyrene nanoporous template were treated with $\mathrm{CF}_{4} / \mathrm{O}_{2}$ plasma $\left(\mathrm{O}_{2} / \mathrm{CF}_{4}=20 \mathrm{sccm} / 20 \mathrm{sccm}, 20 \mathrm{mtorr}\right.$, $50 \mathrm{~W}, 17 \mathrm{~s})$ to remove the remnant PMMA cylinder cores and etch the Si substrate slightly. The substrates were cleaned with BHF (10 s) and then rinsed with DI water to remove the oxide layer on the exposed $\mathrm{Si}$ surface (within the nanopores). A thin Au film $(20 \mathrm{~nm})$ was deposited over the PS nanoporous template, and the PS nanoporous template was lifted-off upon sonication in toluene. This lift-off process leaves uniformly sized Au nanoparticles arrayed in a hexagonal lattice defined by the pores of the nanoporous PS template. Thus prepared substrates were dipped into $6 \mathrm{M} \mathrm{HF} / 0.6 \mathrm{M} \mathrm{H}_{2} \mathrm{O}_{2}$ aqueous solution for $0-10 \mathrm{~min}$ for $\mathrm{Au}$ catalysed Si etching. After the Au catalysed etching, Au was removed with $\mathrm{Au}$ etchant, and rinsed with DI water.

\section{Fabrication of Si nanowire electrode}

The substrates with the polystyrene nanoporous template were further treated with oxygen plasma $\left(\mathrm{O}_{2} 40 \mathrm{sccm}, 20\right.$ mtorr, $50 \mathrm{~W}$, $15 \mathrm{~s})$ to remove any remnant PMMA cylinder cores. The thin film of $\mathrm{Cr}(20 \mathrm{~nm})$ was deposited over the nanoporous PS template. After the deposition process, the nanoporous PS template was lifted off by sonication in a piranha solution $(20 \mathrm{~min})$. This lift-off process leaves uniformly sized $\mathrm{Cr}$ nanoparticles arranged in a hexagonal lattice defined by the pores of the nanoporous PS template. This hexagonal lattice of $\mathrm{Cr}$ nanoparticles was used an etching mask for the Au-catalyzed Si etching. To prevent the continuous layer of Au film, the exposed Si surface (through the $\mathrm{Cr}$ nanoparticle mask) was slightly etched $(\approx 15 \mathrm{~nm})$ by using reactive ion etching (RIE) $\left(\mathrm{SF}_{6} / \mathrm{C}_{4} \mathrm{~F}_{8}=10 \mathrm{sccm} / 20 \mathrm{sccm}, 100 \mathrm{~W} /\right.$ $100 \mathrm{~W}, 5 \mathrm{mtorr}, 20 \mathrm{~s}$ ), and the etched $\mathrm{Si}$ surface was cleaned using a piranha solution and BHF for $10 \mathrm{~min}$ and $20 \mathrm{~s}$, respectively. Then a thin Au catalyst film $(8 \mathrm{~nm})$ was deposited on the 
$\mathrm{Si}$ surface. The surfaces with the deposited $\mathrm{Au}$ catalyst were dipped into a $6 \mathrm{M} \mathrm{HF} / 0.6 \mathrm{M} \mathrm{H}_{2} \mathrm{O}_{2}$ aqueous solution for 0-20 min. The Au catalyst in contact with the Si surface (defined by the $\mathrm{Cr}$ nanoparticle mask) etched the Si vertically through the Cr-nanoparticle etching mask leading to a dense, vertical Si nanowire array. After the metal-assisted etching, the $\mathrm{Cr} / \mathrm{Au}$ was removed with the $\mathrm{Cr} / \mathrm{Au}$ etchant and rinsed with DI water.

\section{Platinum deposition}

The prepared low-doped (doping concentration: $\approx 10^{15} \mathrm{~cm}^{-3}$ ) ptype silicon nanowire/nanomesh electrodes were doped with with boron at the bottom and phosphorous at the top using solid targets in a tube furnace at $1000^{\circ} \mathrm{C}$ for $20 \mathrm{~min}$ (doping concentration: $\approx 10^{20} \mathrm{~cm}^{-3}$, doping thickness: $\approx 2 \mu \mathrm{m}$ ). The silicon nanowire/nanomesh was completely doped with phosphorous using this process. Pt deposition on the prepared Si nanomesh/nanowire electrodes was accomplished using a galvanic displacement reaction in which $\mathrm{Si}$ is oxidized and $\mathrm{Pt}$ is reduced onto the electrode surface. The solution used for $\mathrm{Pt}$ deposition contained 0.05-1 $\mathrm{mm} \mathrm{Na}_{2} \mathrm{PtCl}_{6} \cdot 6 \mathrm{H}_{2} \mathrm{O}$ and $0.5 \mathrm{M} \mathrm{HF}$. The electrodes were immersed in the solution for $10 \mathrm{~min}$ at room temperature without stirring of the solution. After Pt deposition, a thin Al film $(100 \mathrm{~nm})$ was deposited on the back side of electrodes by using electron-beam evaporation at a base pressure of approximately $2 \times 10^{-6}$ torr. The Al-deposited electrodes were connected with a metal wire for measurements, and the electrodes were completely sealed with epoxy with the exception of a working area of approximately $0.5 \mathrm{~cm}^{2}$.

\section{Photoelectrochemical activity measurements}

All of the photoelectrochemical measurements were performed in aqueous $0.5 \mathrm{M} \mathrm{K}_{2} \mathrm{SO}_{4}$ solution, and the solution was adjusted to $\mathrm{pH} 1$ with $\mathrm{H}_{2} \mathrm{SO}_{4}$. The measurements were performed by using a three-electrode configuration utilizing a $\mathrm{Pt}$ wire as a counter electrode and a $\mathrm{Ag} / \mathrm{AgCl}$ electrode as the reference electrode. The potential was swept from positive to negative potentials with a scan rate of $50 \mathrm{mV} \mathrm{s}^{-1}$. Data was measured with versa STAT 3 (Princeton Applied Research), and the collected data were shifted on the potential axis so that the potential of the reversible hydrogen electrode (RHE) was zero. During the measurements, the cells were illuminated using a tungsten-halogen lamp with the intensity of $0-70 \mathrm{~W} \mathrm{~cm}^{-2}$ at the working electrode, and the light intensity was calibrated by using a Si photodiode.

\section{Absorbance and Quantum efficiency measurement}

Absorbance measurements were obtained using an Oriel $150 \mathrm{~W}$ Xe arc lamp (Newport) and a quarter-turn single-grating monochromator (Newport). Sample measurements were recorded using chopped illumination $(15 \mathrm{~Hz})$, and a quartz beam splitter was used to simultaneously record the light output intensity with a separate Si photodiode (Newport) to adjust for fluctuations in lamp intensity. The wavelength-dependent external quantum yield values were measured at $-0.8 \mathrm{~V}$ vs. RHE for each device, and the absolute photocurrents were measured using a digital PAR 273 potentiostat. The output current signal was connected to a Stanford Instruments SR830 lock-in amplifier, and the output signals from the lock-in amplifier and the reference $\mathrm{Si}$ photodiode were fed into a computer controlled by custom-written LabVIEW software.

\section{Acknowledgements}

We acknowledge financial support from the National Science Foundation (grant CBET-1066447), and the Air Force Office of Scientific Research (AFOSR) (grant FA9550-11-1-0017). This work used the Lurie Nanofabrication Facility at the University of Michigan, a member of the National Nanotechnology Infrastructure Network funded by the National Science Foundation.

Keywords: block copolymers - hydrogen production nanomaterials $\cdot$ photoelectrolysis $\cdot$ silicon

[1] M. S. Dresselhaus, I. L. Thomas, Nature 2001, 414, 332.

[2] L. Schlapbach, A. Zuttel, Nature 2001, 414, 353.

[3] A. I. Hochbaum, P. D. Yang, Chem. Rev. 2010, 110, 527.

[4] M. Grätzel, Nature 2001, 414, 338.

[5] M. G. Walter, E. L. Warren, J. R. McKone, S. W. Boettcher, Q. X. Mi, E. A. Santori, N. S. Lewis, Chem. Rev. 2010, 110, 6446.

[6] A. Kudo, Y. Miseki, Chem. Soc. Rev. 2009, 38, 253.

[7] S. W. Boettcher, J. M. Spurgeon, M. C. Putnam, E. L. Warren, D. B. Turner-Evans, M. D. Kelzenberg, J. R. Maiolo, H. A. Atwater, N. S. Lewis, Science 2010, 327, 185.

[8] S. Y. Reece, J. A. Hamel, K. Sung, T. D. Jarvi, A. J. Esswein, J. J. H. Pijpers, D. G. Nocera, Science 2011, 334, 645.

[9] Y. Hou, B. L. Abrams, P. C. Vesborg, M. E. Bjorketun, K. Herbst, L. Bech, A. M. Setti, C. D. Damsgaard, T. Pedersen, O. Hansen, J. Rossmeisl, S. Dahl, J. K. Norskov, I. Chorkendorff, Nat. Mater. 2011, 10, 434.

[10] S. W. Boettcher, E. L. Warren, M. C. Putnam, E. A. Santori, D. Turner-Evans, M. D. Kelzenberg, M. G. Walter, J. R. McKone, B. S. Brunschwig, H. A. Atwater, N. S. Lewis, J. Am. Chem. Soc. 2011, 133, 1216.

[11] Q. Ding, F. Meng, C. R. English, M. Caban-Acevedo, M. J. Shearer, D. Liang, A. S. Daniel, R. J. Hamers, S. Jin, J. Am. Chem. Soc. 2014, 136,8504 .

[12] M. T. Mayer, C. Du, D. Wang, J. Am. Chem. Soc. 2012, 134, 12406.

[13] X. Li, Y. Xiao, J. H. Bang, D. Lausch, S. Meyer, P. T. Miclea, J. Y. Jung, S. L. Schweizer, J. H. Lee, R. B. Wehrspohn, Adv. Mater. 2013 $25,3187$.

[14] E. L. Warren, J. R. McKone, H. A. Atwater, H. B. Gray, N. S. Lewis, Energy Environ. Sci. 2012, 5, 9653.

[15] B. M. Kayes, H. A. Atwater, N. S. Lewis, J. Appl. Phys. 2005, 97, 114302.

[16] M. J. Price, S. Maldonado, J. Phys. Chem. C 2009, 113, 11988.

[17] B. Z. Tian, X. L. Zheng, T. J. Kempa, Y. Fang, N. F. Yu, G. H. Yu, J. L. Huang, C. M. Lieber, Nature 2007, 449, 885.

[18] L. Hu, G. Chen, Nano Lett. 2007, 7, 3249.

[19] J. Zhu, Z. Yu, G. F. Burkhard, C. M. Hsu, S. T. Connor, Y. Xu, Q. Wang, M. McGehee, S. Fan, Y. Cui, Nano Lett. 2009, 9, 279.

[20] J. Y. Kwon, D. H. Lee, M. Chitambar, S. Maldonado, A. Tuteja, A. Boukai, Nano Lett. 2012, 12, 5143.

[21] S. J. Jeong, G. D. Xia, B. H. Kim, D. O. Shin, S. H. Kwon, S. W. Kang, S. O. Kim, Adv. Mater. 2008, 20, 1898.

[22] D. H. Lee, W. J. Lee, S. O. Kim, Nano Lett. 2009, 9, 1427.

[23] D. Zschech, D. H. Kim, A. P. Milenin, R. Scholz, R. Hillebrand, C. J. Hawker, T. P. Russell, M. Steinhart, U. Gosele, Nano Lett. 2007, 7, 1516.

[24] J. Y. Cheng, A. M. Mayes, C. A. Ross, Nat. Mater. 2004, 3, 823.

[25] E. M. Hicks, S. L. Zou, G. C. Schatz, K. G. Spears, R. P. Van Duyne, L. Gunnarsson, T. Rindzevicius, B. Kasemo, M. Kall, Nano Lett. 2005, 5, 1065 .

[26] S. Y. Chou, P. R. Krauss, P. J. Renstrom, Science 1996, 272, 85.

[27] Z. P. Huang, N. Geyer, P. Werner, J. de Boor, U. Gosele, Adv. Mater. 2011, 23, 285. 
[28] K. Q. Peng, A. J. Lu, R. Q. Zhang, S. T. Lee, Adv. Funct. Mater. 2008 $18,3026$.

[29] A. I. Hochbaum, R. K. Chen, R. D. Delgado, W. J. Liang, E. C. Garnett, M. Najarian, A. Majumdar, P. D. Yang, Nature 2008, 451, 163.

[30] J. J. H. Pijpers, M. T. Winkler, Y. Surendranath, T. Buonassisi, D. G. Nocera, Proc. Natl. Acad. Sci. USA 2011, 108, 10056.

[31] I. Lombardi, S. Marchionna, G. Zangari, S. Pizzini, Langmuir 2007, $23,12413$.
[32] F. Amano, D. Li, B. Ohtani, Chem. Commun. 2010, 46, 2769.

Received: July 4, 2014

Revised: September 2, 2014

Published online on September 18, 2014 